\title{
Multifractal Measure of the Solar Magnetic Field
}

Article in The Astrophysical Journal · October 1993

DOI: $10.1086 / 173360$

CITATIONS

87

3 authors:

John K. Lawrence

California State University, Northridge

147 PUBLICATIONS 1,092 CITATIONS

SEE PROFILE

Ana Cadavid

California State University, Northridge

98 PUBLICATIONS 878 CITATIONS

SEE PROFILE
READS

28

Alexander Ruzmaikin

California Institute of Technology

400 PUBLICATIONS $\quad \mathbf{5 , 0 8 0}$ CITATIONS

SEE PROFILE

All content following this page was uploaded by Ana Cadavid on 26 September 2014. 
ThE AstrophysiCal Journal, 417:805-811, 1993 November 10

(C) 1993. The American Astronomical Society. All rights reserved. Printed in U.S.A.

\title{
MULTIFRACTAL MEASURE OF THE SOLAR MAGNETIC FIELD
}

\author{
J. K. Lawrence, A. A. Ruzmaikin, and A. C. Cadavid \\ San Fernando Observatory, Department of Physics and Astronomy, California State University, \\ Northridge, 18111 Nordhoff Street, Northridge, CA 91330 \\ Received 1993 March 4; accepted 1993 May 13
}

\begin{abstract}
We analyze high-resolution, digital, photoelectric images of solar photospheric magnetic fields. The line-ofsight fields are found to scale in a self-similar way with resolution and thus can be expressed in the form of a signed multifractal measure. The scaling properties of the measure are used to extrapolate field integrals, such as moments of the magnetic field, below resolvable limits. The scaling of the field moments is characteristic of highly intermittent fields. We suggest that the quiet-Sun photospheric fields are generated by local dynamo action based on random convective motions at high magnetic Reynolds number. The properties of active region images are determined by the presence of fields generated by the global, mean field dynamo.
\end{abstract}

Subject headings: Sun: magnetic fields

\section{INTRODUCTION}

Magnetic flux in the solar photosphere is concentrated in intense (1000-2000 G), discrete flux tubes separated by essentially field-free plasma (Howard \& Stenflo 1972; Stenflo 1989). The diameter of the basic photospheric flux tube is thought to be less than $\sim 0$ ". 2 , beyond the resolution of any Earth-based instrument (but see Keller 1992). Efforts have been made to model flux tube structures on the basis of observed spectral line profiles (Stenflo 1989). Solar physicists, however, still largely describe solar magnetic structures in qualitative, morphological terms, such as "network" or "intranetwork" fields, "magnetic knots" and the like (Zwaan 1987). A more directly physical approach is needed.

Photospheric magnetism is controlled by the uniquely large magnetic Reynolds number $\left(R_{m} \approx 10^{8}\right)$ characterizing the plasma flow. Because $R_{m}$ is so large, the field is highly intermittent, even at small scales (Zel'dovich et al. 1987). The distribution density of observed flux values is strongly nonGaussian. Although this intermittence can be qualitatively modeled (Meneguzzi, Frisch, \& Pouquet 1981), quantitative understanding of the intermittence in terms of the MHD equations is unworkable. The necessary computer simulations are beyond present day technology.

One avenue of progress lies in the study, not of the smallest magnetic structures, but rather in the way in which the field distribution changes when observed at varying levels of coarseness. This range includes resolvable scales. In our case, the data will be digital, photometric images of line-of-sight magnetic field in the solar photosphere. The primary data, obtained at the San Fernando Observatory (SFO), are made at spatial resolution $\geq 1^{\prime \prime}$ over active region (AR) sized areas. These data will be supplemented by two higher resolution ( $\approx 0$.'5) Lockheed/La Palma magnetograms.

In recent years, a number of modern procedures have been developed in other fields of physics for handling scaling properties in disordered media (Frisch \& Parisi 1985; Bunde \& Havlin 1991; Zel'dovich, Ruzmaikin, \& Sokoloff 1990). These techniques include fractal and multifractal geometries (Mandelbrot 1982; Meneveau \& Sreenivasan 1991; Evertsz \& Mandelbrot 1992). Use is now being made of some of these methods in astrophysics (Heck \& Perdang 1991). Some attempts have been made to apply fractal geometry to solar photospheric field motions (Lawrence 1991; Schrijver et al. 1992; Lawrence \& Schrijver 1993). A number of studies have examined the fractal dimensions of sets of magnetogram image pixels which contain line-of-sight magnetic flux (Greimel et al. 1990; Tarbell et al. 1990; Ruzmaikin, Sokoloff, \& Tarbell 1991; Balke et al. 1993). The existence of a fractal dimension for a set signifies a self-similar geometry of its elements over a range of scales. Thus, the arrangement of magnetic pixels in an image is statistically unchanged when we zoom in and out. We emphasize the connection between fractal geometry, self-similarity and power-law scaling relations.

We shall seek to characterize photospheric magnetic fields in multiscale terms. One benefit of doing so is that the field distribution and its measure scale with varying resolution. If the photospheric fields are multifractal in nature, then we obtain a scaling property which can extrapolate physical conditions below observed resolution limits.

In $\S 2$ we describe the observational data to be analyzed. In $\S 3$ we introduce the concept of a multifractal measure and apply this to observed line-of-sight fields. In $\S 4$ we consider the scaling properties of Gaussian random variables and show where these appear in the data. Section 5 is concerned with properties of signed measures. In $\S 6$ we use the scaling properties of the measure to examine field moments and connect them to the intermittent nature of the field. Section 7 makes connection with the work of others using moments of positive measures. Results are discussed and conclusions drawn in $\S 8$.

\section{OBSERVATIONS}

The primary data used are two-dimensional, highresolution, digital, photometric images of line-of-sight magnetic field. The data are obtained with the SFO $28 \mathrm{~cm}, \mathrm{f} / 20$ vacuum telescope and vacuum spectroheliograph (SHG) operated in the video spectra-spectroheliograph (VS $\left.{ }^{2} \mathrm{HG}\right)$ mode. The VS ${ }^{2} \mathrm{HG}$ is described by Chapman \& Walton (1989) and Lawrence, Chapman, \& Walton (1991). Other major instruments are described in Mayfield et al. (1969).

The images are derived from effective data "cubes" representing two spatial dimensions on the Sun and one spectral dimension. The observed spectral range includes the profile of the solar Fe I line at $6302.5 \AA(g=2.5)$, a Telluric $\mathrm{O}_{2}$ line at $6302.0 \AA$, and a blended telluric $\mathrm{O}_{2}$ line at $6302.8 \AA$. The 
spectral scale of the image "cubes" is $8.8 \mathrm{~m} \AA$ pixel $^{-1}$. The E-W spatial scale is 0.46 pixel $^{-1}$. The N-S spatial scale is similar but depends on the SHG scanning rate.

The cubes are reduced to spatial maps by carrying out operations on the data along the spectral dimension. The cubes are obtained in both left- and right-handed circular polarizations, which can be converted to Stokes $I$ and $V$ line profiles. Then, a map of line-of-sight magnetic field (equivalent to net flux integrated over the pixel area) is generated from the first moment of the Stokes $V$ profile about the line center, divided by the equivalent width of the $I$ profile. The estimated noise is $\pm 15 \mathrm{G}$. The spatial resolution is seeing limited at more than $1^{\prime \prime}$, and the field of view is about $200^{\prime \prime}$ for the $28 \mathrm{~cm}$ telescope. A magnetic image made with the $28 \mathrm{~cm}$ telescope under good seeing conditions is shown in Figure 1 (Plate 28).

Other data used are two very high-resolution images made with the SOUP tunable filter instrument at the Swedish Solar Observatory, La Palma (Title et al. 1989). Under the best seeing conditions, these can produce magnetograms with spatial resolution finer than 0.5 . The images are composed of $512 \times 512$ pixels of scale 0.14 pixel $^{-1}$ and field of view $85^{\prime \prime} \times 85^{\prime \prime}$. The chief advantage of these data is their unparalleled spatial resolution.

\section{MULTIFRACTAL MEASURES}

The procedure used for determining a fractal dimension of the solar field is the "box counting" method (Mandelbrot 1982). Let a Euclidean plane (our magnetic image, which represents some projection of the solar surface) contain a geometrical object (the set of pixels in the image containing magnetic field). We cover the image with small, square boxes or cells of side $\epsilon$. Then we count the number of boxes $N(\epsilon)$ covering the set, that is, containing some field. As the size $\epsilon$ of the boxes is varied, the number will obey a power law

$$
\begin{gathered}
N(\epsilon) \propto \epsilon^{-D} \\
\ln N(\epsilon)=D \ln (1 / \epsilon)+C .
\end{gathered}
$$

If the geometric object is a line, then we find $D=1$; if it fills the plane with uniform density, then we find $D=2$. For solar magnetic fields, we find a "fractal" dimension $1<D<2$. The variable $D$ does not refer to a particular scale, but rather governs the self-similarity as the scale changes.

The membership of a pixel in the fractal set is determined only by the presence or absence of flux meeting some criterion (like $|H|>H$ threshold) and not by its amount. The measured fractal dimensions of the fields, however, were found to be dependent on the value of the threshold criterion (Ruzmaikin et al. 1991). This indicates the presence of a geometrical structure more complicated than a simple fractal.

We now generalize the fractal concept by adding a "measure" defined by the values of the net magnetic flux through each pixel. When, along with its supporting set, a measure also displays self-similarity, the measure is said to be multifractal (Frisch \& Parisi 1985; Evertsz \& Mandelbrot 1992). Fields in line-of-sight magnetic images made at the San Fernando Observatory, and in Lockheed-La Palma magnetograms, have this property.

Associating a measure with a supporting set is analogous to taking into account the values of coins in our pockets rather than only their number. Measure is additive; the measure of the union of disjoint subsets is the sum of the measures of the subsets. This means that the value of a collection of coins is the sum of the total value of the pennies plus the total value of the dimes, and so on. Another example would be associating with the set of outcomes of throwing two dice (the integers $2 \ldots 12$ ) a measure giving the probability for each outcome (e.g., $\mu_{2}=$ $1 / 36$ or $\mu_{7}=1 / 6$ ). The measure of the outcome subset " 2 or 7 " is the sum $\mu_{2}+\mu_{7}=7 / 36$. A probability measure has the property $\Sigma \mu_{i}=1$.

The concept of a measure is closely related to the process of integration. The two-dimensional Lebesgue measure $d \mu_{i}$ of any subset of the Euclidean plane is just its area. The ordinary Riemann integral of a function $f(x, y)$ is

$$
\Sigma f\left(x_{i}, y_{i}\right) d \mu_{i}=\int f(x, y) d x d y .
$$

The meaningfulness of this integral depends on $f(x, y)$ being smooth on the scale of $d \mu$. In this paper we will find that the solar photospheric magnetic field is singular on observable scales, and perhaps on much smaller scales as well, so that using Riemannian integrals to calculate such quantities as field energy may be inappropriate. We will suggest an alternate measure for use in magnetic integrals. The self-similarity of this measure will allow us to extrapolate their properties below observable levels.

First, we select an $L \times L$ pixel image array whose elements are the values of the line-of-sight field strength $H_{i}$ in the $i$ th pixel. This constitutes a "signed measure" (Halmos 1962, chap. 6). This is converted to a positive definite measure similar to a probability measure by calculating the normalized absolute value of the field: $\mu_{i} \equiv\left|H_{i}\right| / \Sigma\left|H_{i}\right|$. Because magnetic flux is an extensive quantity while magnetic field is a flux density, it is more appropriate to consider the measure $\mu_{i}$ to represent absolute values of the fraction of the net $f u x$ through the $i$ th pixel. In the limit of infinite spatial resolution the values of field strength and flux divided by area of the resolution element coincide. At coarse-grained levels they do not. The measure we have defined is closely related to the concept of the magnetic "filling factor" which represents the portion of a pixel or resolution element occupied by flux tubes.

Usually $L=256$ pixels (sometimes 240 pixels). The smallest possible box, of course, is a single square pixel; we define its size to be $\epsilon_{0}=1 / L \ll 1$. The "coarse-grained" measure $\mu_{i}(\epsilon)$ in boxes of side $\epsilon_{n}=S_{n} / L=2^{n-8}, n=0,1,2 \ldots$ is calculated by a renormalization procedure in which the oppositely directed line-of-sight components are allowed to cancel within the box before the absolute value is used to compute a normalized, positive definite measure $\mu_{i}(\epsilon)$, similar to a probability measure. The coarse-graining imitates degraded observational resolution. For consistency with observational data we set to zero the measure in any box equivalent to a flux density less than 1 gauss.

We hypothesize that the measure is self-similar and thus multifractal. Then the measure, and its distribution, must scale with a power-law dependence on $\epsilon$. The scaling "Hölder exponent" $\alpha$ gives the presumed self-similar scaling of the measure in the $i$ th box when observed at different resolutions $\epsilon$ :

$$
\mu(\epsilon)=\epsilon^{\alpha} \text {. }
$$

It is easy to compute a value for $\alpha_{i}$ in each box for a given scale $\epsilon$ :

$$
\alpha_{i}=\ln \mu_{i}(\epsilon) / \ln \epsilon
$$

If the measure $\mu$, representing magnetic flux, scales like $\epsilon^{\alpha}$, then the line-of-sight magnetic field, which is the flux density, must 


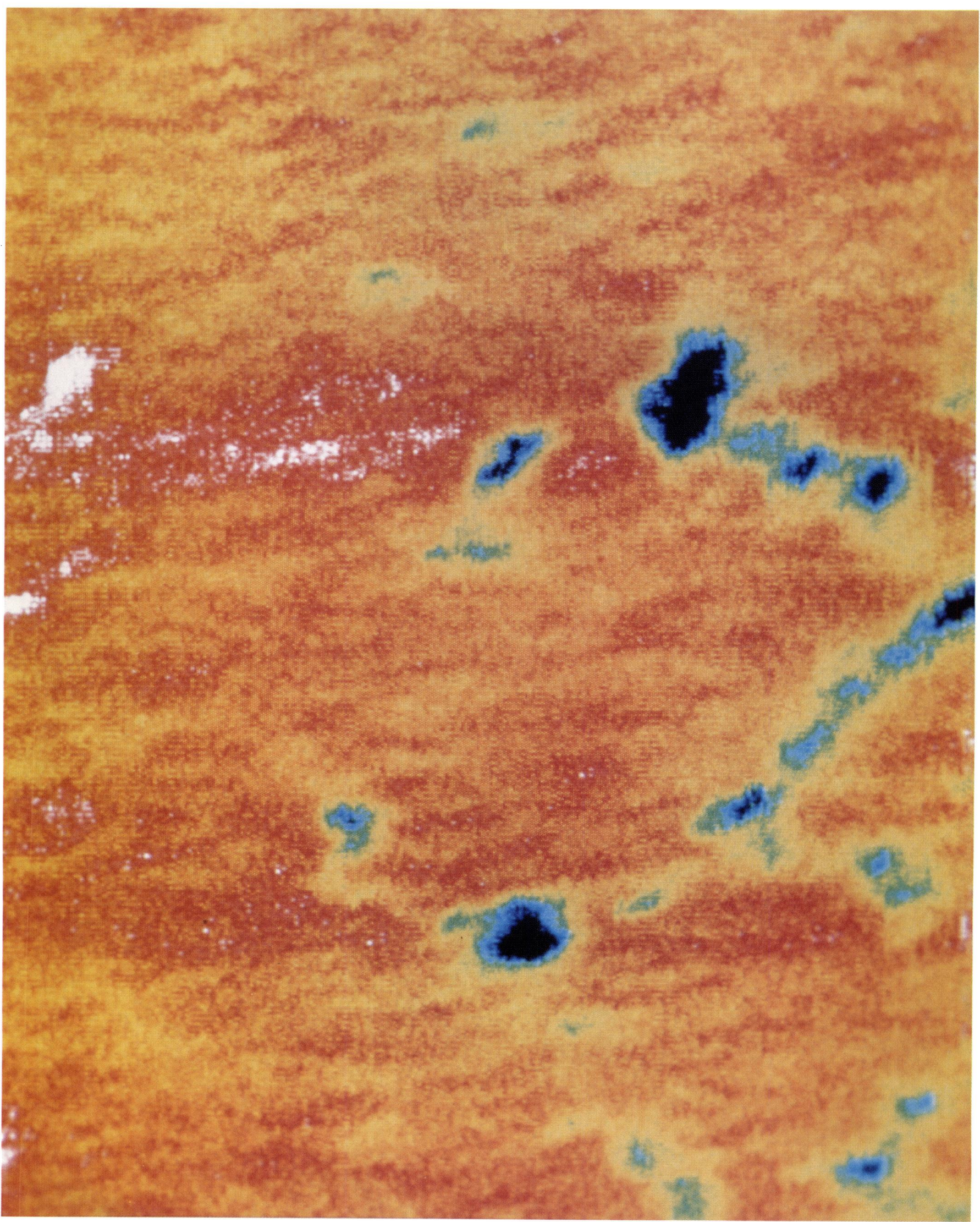

FIG. 1-SFO false color line-of-sight magnetic image of QS at heliocentric angle $\sim 23^{\circ}$ on 21 September 1992 at UT 18:16:00. The field of view is 375 pixels $=173^{\prime \prime} \mathrm{E}-\mathrm{W}$ and 263 pixels $=121^{\prime \prime} \mathrm{N}-\mathrm{S}$. The magnetic field varies from $+135 \mathrm{G}$ (white) to $-279 \mathrm{G}$ (black). Noise is $\sim 15 \mathrm{G}$.

LaWrence, Ruzmaiken, \& CADAVID (see 417, 806)

(C) American Astronomical Society • Provided by the NASA Astrophysics Data System 
scale like $\epsilon^{\alpha-2}$. Thus if $\alpha<2$, the field strength is singular. For this reason $\alpha_{i}$ is also referred to as the "singularity strength" in the $i$ th box.

To characterize the distribution of the measure for each coarse-graining scale $\epsilon$, we count the number $d N(\alpha, \epsilon)=$ $n(\alpha, \epsilon) d \alpha$ of boxes with singularity strength $\alpha$ in bins of width $d \alpha$. For a multifractal measure we expect the density $n(\alpha, \epsilon)$ to scale according to a power law

$$
n(\alpha, \epsilon)=\phi(\epsilon) \epsilon^{-f(\alpha)}
$$

and

$$
f(\alpha)=-\ln [n(\alpha, \epsilon) / \phi(\epsilon)] / \ln \epsilon .
$$

The slowly varying function $\phi(\epsilon)$ is required by the normalization condition $\int n(\alpha, \epsilon) \mu(\alpha) d \alpha=1$. It reduces to a power series in $1 / \ln (1 / \epsilon)$ :

$$
\phi(\epsilon)=[\ln (1 / \epsilon)]^{-1 / 2}\left[1+C_{1} / \ln (1 / \epsilon)+\ldots\right]
$$

(Meneveau \& Sreenivasan 1989). The quantity $C_{1}$ is a constant independent of $\epsilon$; it depends on the curve $f(\alpha)$ and its derivatives. We will treat $C_{1}$ as a free parameter and drop higher order corrections.

Our hypothesis that the measure $\mu$ is self-similar, and thus multifractal, is justified if the derived $f(\alpha)$ collapse to a single curve in the limit of small $\epsilon$. Figure 2 shows plots of $f(\alpha)$ versus $\alpha$ for five values of $\epsilon_{n}=S_{n} / L$ for $S_{n}=1,2,4,8,16$. These are derived from a single $L=256$ sample of the SFO magnetic image in Fig. 1 of the quiet sun (QS) photosphere far from any active regions. We note that seeing limits the actual resolution to $S_{n} \geq 2$ and that poor counting statistics limit the accuracy of the curves for $S_{n} \geq 16$. In all figures the counting statistics for the larger box sizes can be improved by applying a Monte Carlo method that will be used in future work. The counting statistics are better in Figure 3, which shows $f(\alpha)$ versus $\alpha$ curves for five values of $S_{n}=2,4,8,16,32$ representing aver-

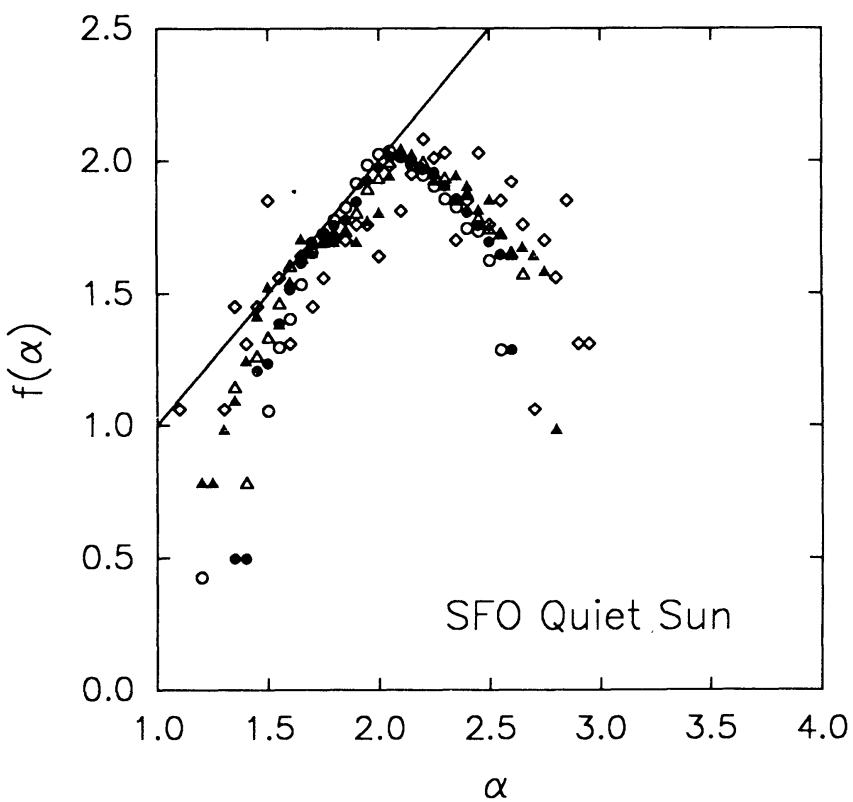

FIG. 2. $-f(\alpha)$ vs. $\alpha$ for a $256 \times 256$ pixel $^{2}$ sample of the 1992 September 21 image in Fig. 1. The straight line is the bisector $f(\alpha)=\alpha$. The coarse-graining scales $S_{n}$ are (open circles) 1 pixel; (filled circles) 2 pixels; (open triangles) 4 pixels; ( filled triangles) 8 pixels; (open diamonds) 16 pixels.

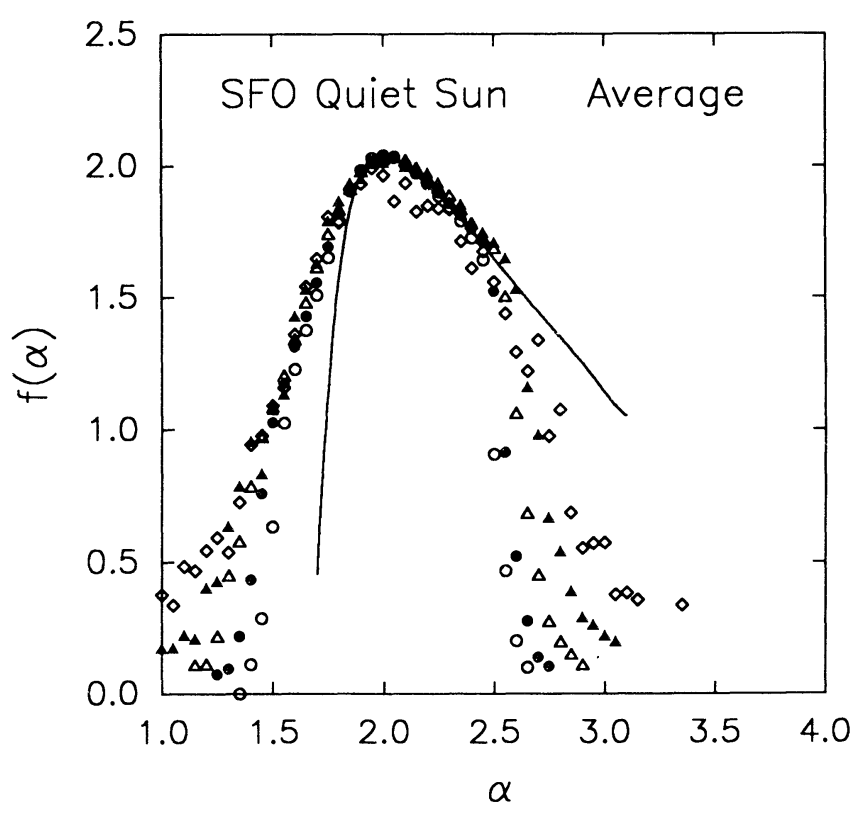

FIG. 3. - $f(\alpha)$ vs. $\alpha$ averaged over $60256 \times 256$ pixel $^{2}$ samples of 30 SFO QS line-of-sight magnetic images like that of Fig. 1 made on 1992 September 19 and 21. Heliocentric angles range over $0^{\circ} \leq \theta \leq 60^{\circ}$. The coarse-graining scales $S_{n}$ are (open circles) 2 pixels; (filled circles) 4 pixels; (open triangles) 16 pixels; (open diamonds) 32 pixels. The solid line is the analytical curve for Gaussian random variables for $S_{n}=2, p=0.9$, and $m=0.7$.

ages over $60 L=256$ samples from 30 QS images. In Figures 2 and 3 the choice $C_{1}=-1$ brings the $f(\alpha)$ curves into close agreement over more than a decade of coarse-graining scales.

Figure 4 shows $f(\alpha)$ curves for $S_{n}=1,2,4,8,16$ for two samples of an image containing a magnetic active region. A choice of $C_{1}=-1.5$ brings the left-hand portion of these curves into good correspondence. We will argue below that the nonmatching secondary maxima on the right-hand sides of these curves represent the presence of a Gaussian random variable component, such as noise, in the data. Figure 5 is an enlargement of Figure 4 curves for $S_{n}=1,2,4,8$. In the SFO images the individual pixel scale is 0.46 , but resolution is limited by seeing at $\geq 1^{\prime \prime}$. We find that the solar field distribution is self-similar to the limit of this resolution. This limit can be extended somewhat by analysis of magnetograms made at the Swedish Solar Observatory at La Palma with the Lockheed SOUP instrument. Two of the finest resolution images of active regions, with individual pixel size 0 ". 14 and resolution $\approx 0$ ".5, have been kindly provided to us for analysis. Figure 6 shows average $f(\alpha)$ curves for four samples of one of the ARs. For clarity we show only the cases $S_{n}=1,2,4,8$. For $C_{1}=$ -1.5 the $f(\alpha)$ curves match closely on the left-hand side while the right hand sides indicate a Gaussian random variable component like that in the SFO image of Figures 4 and 5. The self-similarity of the solar field distribution thus appears to extend to the limit of resolution at $\sim 0$ ".5. Given the very large magnetic Reynolds number of the photospheric plasma, the self-similar intermittence of the fields may extend to much smaller scales. No cutoff scale is indicated by observational data. Its possible value may range from $\approx 0$ ".0014 to $\approx 0$ ". 14 based on theoretical considerations.

Although $f(\alpha)$ itself is regular, it is sometimes called the "singularity spectrum" of the measure because its form summarizes the singularity structure of the flux density. If 


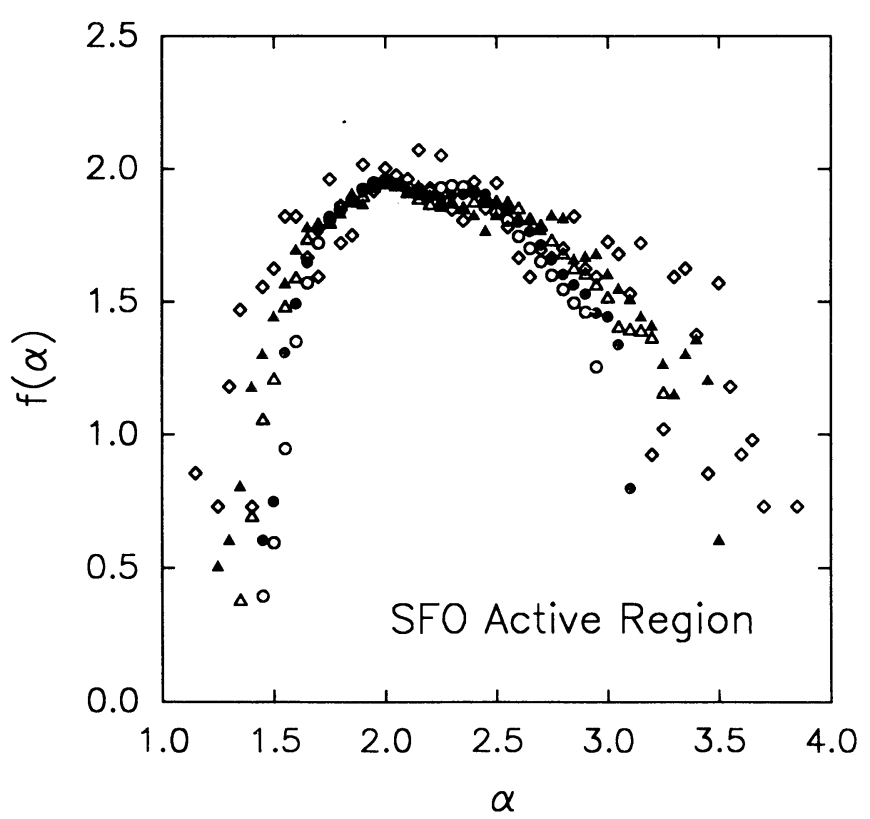

FIG. 4. $-f(\alpha)$ vs. $\alpha$ averaged over two $256 \times 256$ pixel $^{2}$ samples of a 1989 August 17, UT 22:59, SFO line-of-sight magnetic image including active region NOAA 5643 at heliocentric angle $\sim 5^{\circ}$. The coarse-graining scales $S_{n}$ are (open circles) 1 pixel; (filled circles) 2 pixels; (open triangles) 4 pixels; ( filled triangles) 8 pixels; (open diamonds) 16 pixels. Pixel scales are $00^{\prime \prime} 83$ pixel $^{-1} \mathrm{~N}-\mathrm{S}$ and 0.46 pixel $^{-1} \mathrm{E}-\mathrm{W}$.

$f(\alpha)>-\infty$ for $\alpha<2$, then the field is singular as $\epsilon \rightarrow 0$. Comparison of equations (1) and (3) shows that $f(\alpha)$ behaves as a fractal dimension for the subset of boxes with Hölder exponent, or singularity strength, $\alpha$. This is why the measure is called "multifractal" (Frisch \& Parisi 1985). Because all values of $f(\alpha)$ represent the fractal dimension of a subset of the Euclidean plane, the maximum value $f_{m} \leq 2$. This condition is met in Figures 2 to 6 . For $\epsilon \ll 1$, the density $n(\alpha, \epsilon)$ of equation (3) has

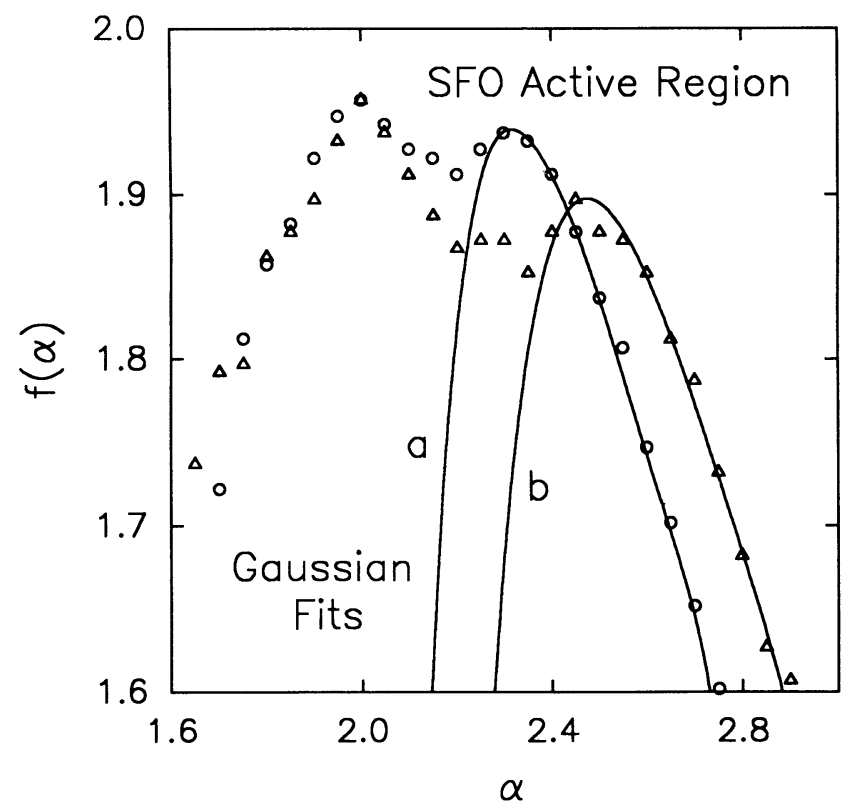

FIG. 5.-Enlargement of Fig. 4 for coarse-graining scales (open circles) 1 and (open triangles) $=4$ pixels. The solid curves are analytical Gaussian random variable fits for $(a) S_{n}=1, p=0.8, m=0.11$ and $(b)$ for $S_{n}=4, p=0.9$, $m=0.10$.

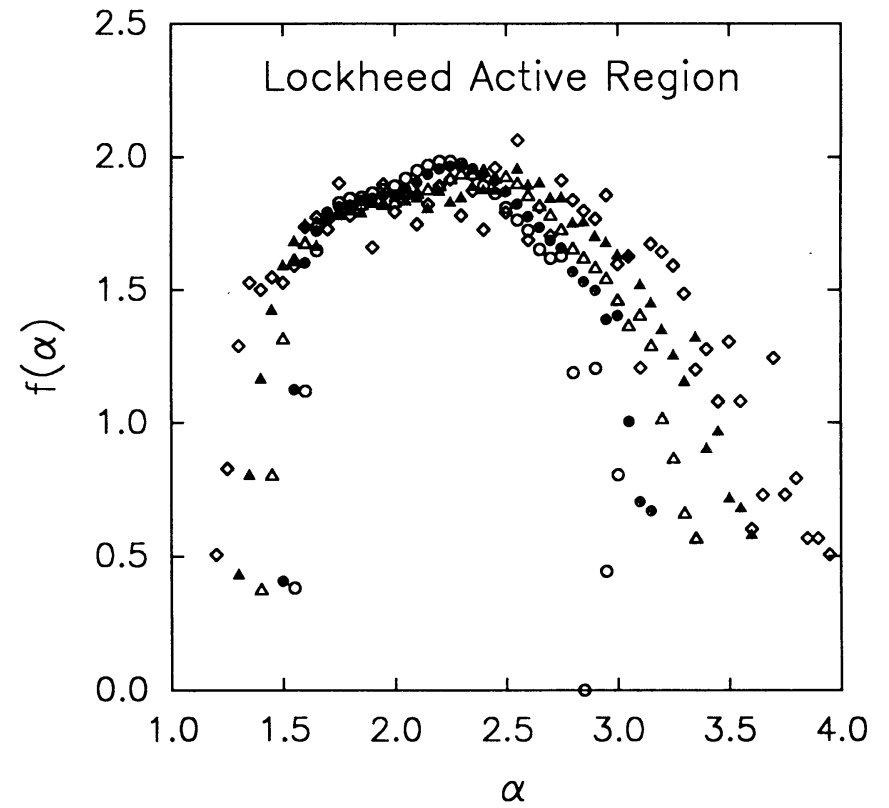

FIG. 6. $-f(\alpha)$ vs. $\alpha$ averaged over four $256 \times 256$ pixel $^{2}$ samples of a $512 \times 512$ pixel $^{2} 1990$ June 6, UT 08:27:19, Lockheed/La Palma line-of-sight magnetogram including NOAA AR No. 6085 at heliocentric angle $\sim 6^{\circ}$. The coarse-graining scales $S_{n}$ are (open circles) 1 pixel; (filled circles) 2 pixels; (open triangles) 4 pixels; (filled triangles) 8 pixels; (open diamonds) 16 pixels. Pixel scale is 0.14 pixel $^{-1}$.

a sharp peak at $\alpha=\alpha_{m}, f=f_{m}$. In the limit, the set of all cells has the fractal dimension $D=f_{m}$; this is the fractal dimension of the supporting set of the multifractal. This corresponds to the fractal dimension in previous studies (Ruzmaikin et al. 1991) in the zero limit threshold.

Summation of the measure is equivalent to integration over $\alpha$ :

$$
\begin{aligned}
\Sigma \mu_{i} & =\int_{-\infty}^{+\infty} n(\alpha, \epsilon) \mu(\alpha, \epsilon) d \alpha \\
& =\int_{-\infty}^{+\infty} \Phi(\epsilon) \epsilon^{-f(\alpha)} \epsilon^{\alpha} d \alpha=1
\end{aligned}
$$

Equation (8) shows how to integrate over the multifractal measure. For $\epsilon \rightarrow 0$, if the summed measure is to be unity (or even finite) then we must have $f(\alpha) \leq \alpha$ everywhere. For the summed measure to be nonzero in the limit we must have $f(\alpha)=\alpha$ at least one point. Thus the "bisector" $f=\alpha$ must be tangent to and above the curve $f=f(\alpha)$ for $\epsilon \rightarrow 0$. This condition is roughly met for the curve of Figure 2 . In practice, however, it is not unusual for $f(\alpha)$ to overshoot the bisector until very small values of $\epsilon(\epsilon \ll 1 / 256)$ are reached. The main contribution to the measure integral comes for $f(\alpha) \approx \alpha$. In this sense we say that the measure is contained in the set with fractal dimension $f(\alpha)=\alpha$.

\section{SCALING OF A GAUSSIAN RANDOM VARIABLE}

To interpret the above singularity spectra, it is instructive to consider an artificial image whose pixel values are chosen at random from a Gaussian distribution centered on zero. This could represent either instrumental noise or a Gaussian process in the small-scale solar magnetic field. The central limit theorem tells us that the sum of many random variables with finite moments approaches a Gaussian random variable. This means that our coarse-graining process described above, when 


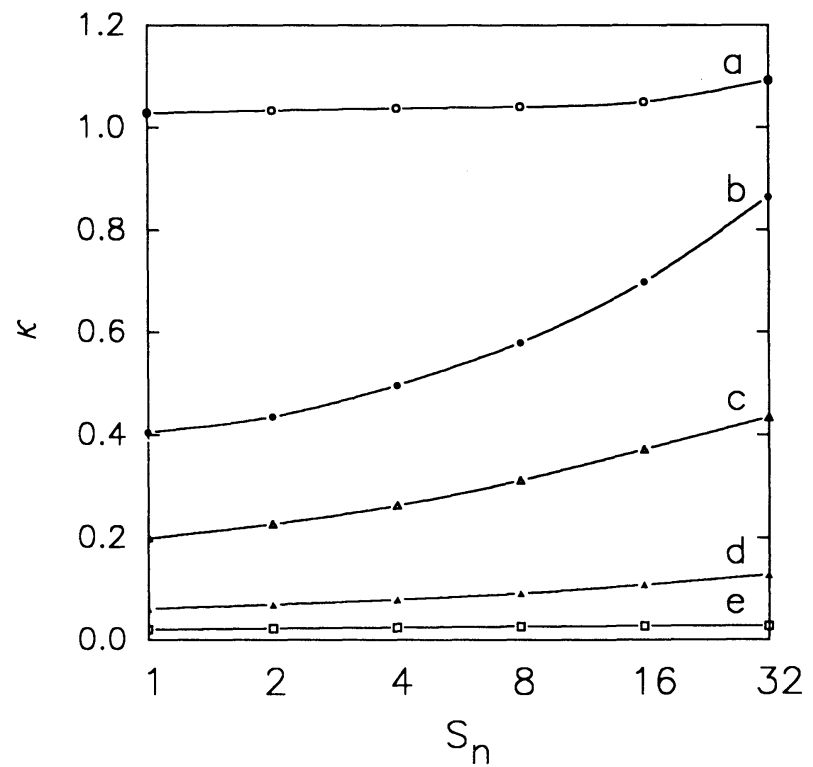

FIG. 7.--Cancellation exponent $\kappa$ vs. coarse graining scale $S_{n}$ for $(a)$ an artificial image composed of a Gaussian random variable centered on zero, $(b)$ The SFO QS image made 1992 September 21 and described in Figs. 1 and 2, (c) for an SFO image of NOAA AR No. 68501991 October 3, $(d)$ for the SFO image of NOAA AR No. 5643 made 1989 August 17 described in Figs. 4 and 5, and $(e)$ the Lockheed/La Palma magnetogram of NOAA AR No. 6085 made 1990 June 6 described in Fig. 6.

applied to the Gaussian random image considered here, will produce another Gaussian random image, and thus a kind of self similarity.

To examine this more precisely, let the Gaussian random image cells at coarse-graining scale $\epsilon$ contain a measure $\mu=|b|$, where $b$ is a random variable with the density

$$
\rho(b, \epsilon)=\frac{p^{2}}{\pi m \epsilon^{4}} \exp \left[-\frac{p^{2} b^{2}}{\pi m^{2} \epsilon^{4}}\right] .
$$

Here $\rho(b, \epsilon) d b$ is the number of cells of side $\epsilon$ with measure in the range $d b$ at $b$. This has the normalization

$$
\int_{-\infty}^{+\infty} d b \rho(b, \epsilon)=\frac{p}{\epsilon^{2}} .
$$

Since $1 / \epsilon^{2}$ is the total number of cells at each coarse-graining level, $p$ is an indicator of the fraction of cells containing $b$. The total measure contained in the random variable $b$ is given by

$$
\int_{-\infty}^{\infty} d b|b| \rho(b, \epsilon)=m .
$$

To convert equation (9) to a singularity spectrum we first calculate a Hölder exponent by replacing $\mu$ with $b$ in equation (3). Then $\rho(b, \epsilon)$ is related to $n(\alpha, \epsilon)$ in equation $(5)$ by $\rho(b, \epsilon) d b=$ $n(\alpha, \epsilon) d \alpha$.

For both $p=1$ and $m=1$, the measure is entirely composed of the random variable, and the singularity spectrum $f(\alpha)$ is independent of $\epsilon$. As $\epsilon \rightarrow 0, f(\alpha) \rightarrow-\infty$ for $\alpha<2$ and $f(\alpha) \rightarrow 4-\alpha$ for $\alpha \geq 2$. For finite $\epsilon, f(\alpha)$ extends to the left and crosses the bisector. The slope remains near -1 on the righthand side. For $p \neq 1$ and/or $m \neq 1$, the $f(\alpha)$ curves are displaced relative to one another for increasing values of $\epsilon$. By appropriate choice of $p$ and $m$, which may vary with $\epsilon$, we can match the analytical singularity spectra for a Gaussian random variable to our data.

Figure 3 shows a fit for $p=0.9$ and $m=0.7$ of the random variable curve to the QS data for the case $S_{n}=2(\epsilon=1 / 128)$. In this case the random variable extends over most of the pixels in the image and contains a large share of the measure. The righthand side of the observed $f(\alpha)$ singularity spectrum is governed entirely by the Gaussian portion of the measure. The left-hand side of $f(\alpha)$ is governed by the long tails of the intermittent field distribution, represented by the stronger fields seen in Figure 1.

In Figure 5 we have fit Gaussian singularity spectra to the curves at two scales. For the $S_{n}=1$ curve we find $p=0.8$ and $m=0.11$. For $S_{n}=4$ we find $p=0.9$ and $m=0.10$. Thus, while the Gaussian fluctuations appear widespread in this AR image, they contain only a small fraction of the measure. Most of the measure is contained in the self-similar active region fields described by the left-hand portion of the singularity spectrum. For the Lockheed AR image in Figure 6, the curves are fit for $p=1$ and $m=0.20,0.16,0.12$, and 0.09 for $S_{n}=1,2,4$ and 8 , respectively.

\section{SIGNED MEASURE AND THE CANCELLATION EXPONENT}

Measures used in physics are generally non-negative, and we have forced our magnetic measure to have this property by using the absolute value of the field after each level of renormalization. However, the actual line-of-sight magnetic field can take both positive and negative values and thus would constitute a signed measure (Halmos 1962). At the level of a single resolution element, measured magnetic fields represent an average over unresolved, small-scale fields, and this may involve significant cancellation of oppositely directed fields. Ott et al. (1992) have pointed out a kind of singularity, peculiar to the theory of signed measures, in which oscillations in sign occur on all scales. As a practical matter, if the oscillations extend below the scale of observational resolution, then the smaller scale oscillations will be averaged away and correct values of the field absolute value or energy cannot be established. Rabin (1992) has presented direct observations of magnetic cancellation in a solar active region.

These authors introduced a "cancellation exponent" to characterize the scaling of the sign oscillations:

$$
\kappa=\lim _{\epsilon \rightarrow 0} \ln \Sigma\left|\mu_{i}\right| / \ln (1 / \epsilon) .
$$

Here we have used the line-of-sight flux itself as the signed measure. As the cell size $\epsilon$ decreases the numerator increases. For a probability density $\Sigma \mu_{i}=1$ and $\kappa$ trivially vanishes. If below some scale $H_{i}$ acquires a smooth density with a single sign, then the numerator will approach a constant, and $\kappa$ will vanish in the limit. For $\kappa$ to remain positive it is necessary that the sum in the numerator grow. This implies cancellation of the signed measure on smaller and smaller scales. Such cancellations are to be expected over the full range of cell sizes in turbulent flow at high magnetic Reynolds number (Ott et al. 1992).

Figure 7 shows the scaling of the cancellation exponent with cell size for a variety of images. For an artificially generated image of pure Gaussian noise $\kappa \rightarrow 1$ for $\epsilon \rightarrow 0$. For the SFO QS image of Figures 1 and $2, \kappa \rightarrow \sim 0.4$. For the SFO AR region of Figures 4 and $5, \kappa \rightarrow \sim 0.05$. For the Lockheed AR image of Figure $6, \kappa \rightarrow \sim 0.02$. This indicates the presence of cancellation in rough proportion to the importance of the Gaussian component of the observed fields. 


\section{SCALING OF MAGNETIC FIELD MOMENTS AND INTERMITTENCY}

The self-similarity of photospheric fields offers the possibility to extrapolate calculations of physical quantities below resolved scales. We use as our example averaged $\left\langle H^{q}\right\rangle$ of the line-of-sight components of the fields, i.e., the field moments.

A model analytical calculation gives some understanding of the problem. The general form of $f(\alpha)$ is cap convex. Let us model $f(\alpha)$ by an inverted parabola:

$$
f(\alpha)=f_{m}-\left(\alpha-\alpha_{m}\right)^{2} / 2 \sigma^{2} .
$$

Here $\alpha_{m}$ is the value of $\alpha$ at which $f$ takes its maximum value $f\left(\alpha_{m}\right)=f_{m}$ and $\sigma$ measures the width of the curve. The number of coarse-grained boxes with singularity strength in bins of width $d \alpha$ at $\alpha$ is

$$
n(\alpha, \epsilon) d \alpha=\phi(\epsilon) \epsilon^{-\left[f_{m}-\left(\alpha-\alpha_{m}\right)^{2} / 2 \sigma^{2}\right]} d \alpha .
$$

Equation (14) can be used to integrate functions of $\alpha$ over the measure. Rather than sum cells by their Cartesian location $x, y$, we sort them in order of their Hölder exponent $\alpha$, and then sum by integrating over $\alpha$.

The net flux through a cell is $\mu=\epsilon^{\alpha}$; the flux density, or magnetic field, is $\epsilon^{\alpha-2}$. The $q$ th power of the field is $\epsilon^{q(\alpha-2)}$. The field and its moments are singular if $\alpha<2$. We sum over a square of unit area

$$
\left\langle H^{q}\right\rangle=\epsilon^{2} \int_{-\infty}^{+\infty} \phi(\epsilon) \epsilon^{-f_{m}+\left(\alpha-\alpha_{m}\right)^{2} / 2 \sigma^{2}+q(\alpha-2)} d \alpha .
$$

The factor $\epsilon^{2}$ is the area of each cell. For small $\epsilon$ most of the contribution to the integral comes when the exponent of $\epsilon$ is minimized. This occurs for $\alpha=\alpha_{m}-q \sigma^{2}$. Thus positive $q$ moments are governed by the $\alpha<\alpha_{m}$ side of the spectrum and the negative moments by the $\alpha>\alpha_{m}$ side. The minimized exponent is

$$
\chi(q)=-\left[f_{m}+\left(q^{2} / 2\right) \sigma^{2}+2 q-q \alpha_{m}-2\right] .
$$

In the limit of small $\epsilon$, the integral diverges if $\chi<0$, vanishes if $\chi>0$, and is finite if $\chi=0$. Since the total measure $\Sigma \mu_{i}$ must be finite, $\chi(1)=0$ which leads to $f_{m}=\alpha_{m}-(1 / 2) \sigma^{2}<\alpha_{m}$ and

$$
\chi(q)=-(q-1)\left[\left(2-f_{m}\right)+\frac{1}{2} q \sigma^{2}\right] .
$$

In the limit that the geometry approaches a pure fractal, with the same Hölder exponent everywhere, $\sigma^{2} \rightarrow 0$, and $f_{m} \rightarrow$ $\alpha_{m} \rightarrow D$, the fractal dimension. Then $\chi(q) \rightarrow-(q-1)(2-D)$. Thus, for a Euclidean geometry, all moments of the field are finite if $q$ is finite. For a fractal dimension $D<2$, the moments for $q>1$ will diverge, while moments for $q<1$ will vanish. The $q=0$ case means that the fraction of cells containing any amount of field approaches zero as the cell size gets smaller. The moment for $q=1$ is finite.

In the multifractal case, $\sigma^{2}>0$, and $f_{m} \leq 2$. Thus for $q>1$, the moment $\left\langle H^{q}\right\rangle$ diverges for small $\epsilon$. If $q=1$ the moment is finite. If $-2\left(2-f_{m}\right) / \sigma^{2}<q<1$ the moment vanishes in the limit. If $q=-2\left(2-f_{m}\right) / \sigma^{2}$ the moment is finite. If $q<$ $-2\left(2-f_{m}\right) / \sigma^{2}$ the moment diverges again.

The "flatness" of the field distribution is $F=\left\langle H^{4}\right\rangle /\left\langle H^{2}\right\rangle^{2}$. For a Gaussian distribution $F=3$. For a Euclidean geometry $\sigma=0, f_{m}=2$, the flatness remains finite. For the multifractal measure $F$ will diverge for small $\epsilon$ as $\chi(4)-2 \chi(2)=-\left[\left(2-f_{m}\right)\right.$ $\left.+4 \sigma^{2}\right]$. This emphasis of larger moments of the field distribution is a defining characteristic of "intermittence" of the field distribution. It corresponds to a non-Gaussian distribution with significant large-field tails.

Consider also the ratio $\left\langle H^{q+1}\right\rangle /\left\langle H^{q}\right\rangle$, which scales with $\epsilon$ according to $\chi(q+1)-\chi(q)=-\left[\left(2-f_{m}\right)+q \sigma^{2}\right]$. This diverges for all $q>0$ if $f_{m}<2$ (for a fractal) and/or $\sigma^{2}>0$ (for a multifractal). In the multifractal case, the rate of divergence increases with increasing moment exponent $q$. This growth also is characteristic of intermittence (Zel'dovich et al. 1990). It is closely related to the multifractality of the field distribution.

\section{POSITIVE MEASURES AND THE METHOD OF MOMENTS}

Our use of a signed measure whose opposing fluxes are permitted to cancel during the rescaling procedure differs from most work on multifractals, which relies on strictly positive measures. An alternative approach to multifractals lies through the scaling properties of moments of the measure (Halsey et al. 1986). Here multifractality means that the $q$ th moment of the measure has a power law scaling with coarsegraining according to

$$
\Sigma \mu^{q} \sim \epsilon^{\tau(q)} .
$$

We can use equation (8) to express this sum as an integral over Hölder exponents:

$$
\Sigma \mu^{q}=\int d \alpha n(\alpha, \epsilon) \epsilon^{\alpha q}=\int d \alpha \varphi(\epsilon) \epsilon^{\alpha q-f(\alpha)} \approx \epsilon^{\tau(q)} .
$$

For small $\epsilon$ the main contribution of the integrand comes at the value of $\alpha$ which minimizes the exponent $\alpha q-f(\alpha)$. This occurs for $q-f^{\prime}(\alpha)=0$ if and only if $f^{\prime \prime}(\alpha)<0$. In this case we find

$$
\alpha=\frac{\partial \tau}{\partial q} ; \quad f(\alpha(q))=q \alpha-\tau(q) .
$$

Thus the variables $\alpha$ and $f(\alpha)$ can be derived from the observed $q$ and $\tau(q)$ by this Legendre transformation. This method has been used by Brandenburg et al. (1992) to study the multifractal properties of magnetic fields in computer simulated magnetoturbulence.

We have successfully used this approach with our data by replacing the measure by its absolute value at the level of individual pixels. This, however, destroys information about the vector nature of the fields, and the coarse-graining procedure no longer imitates the effect of actual observational resolution.

In principle, the method of moments could be used with our renormalization procedure too. However, the cancellation emphasizes noise in the weakest field regions of an image. These are exaggerated in the negative moments of equation (18), and large uncertainties in the scaling appear for negative values of $q$ (which correspond to $\alpha>2$ ). The requirement $f^{\prime \prime}(\alpha)<0$ is not universally met by our observed curves, so the Legendre transformation is not always applicable; see Figures 4 and 6 . We have thus preferred to evaluate the $f(\alpha)$ directly via equations (6) and (7). A method also has been developed (Chhabra et al. 1989) for direct computation of $f(\alpha)$ by means of averages of the moments of the measure like those of equation (18). This bypasses the ambiguous logarithmic correction factors of equation (7), but it requires the condition $f^{\prime \prime}(\alpha)<0$ for its validity. It also produces very regular curves which may conceal details of the singularity spectrum which aid in its interpretation, such as the Gaussian components. For our pur- 
poses we have felt that the most direct histogram method is the best.

\section{DISCUSSION AND CONCLUSIONS}

For quiet-Sun areas the maximum of the singularity spectrum comes very near the critical value $\alpha=2$. For the 1992 September 21 QS case (Fig. 2) and for the summarized QS cases (Fig. 3) the maximum of the curve is $f_{m}=2$, indicating that the supporting set of the multifractal is the whole Euclidean plane. This just means that essentially every pixel contains at least some of the measure. In general, the QS field distributions, which are partly but not entirely Gaussian, conform to multifractal scaling quite well.

We suggest that the magnetic field in the quiet Sun is generated by random convective motions at high magnetic Reynolds number. This produces a structure which resembles in part a Gaussian random variable (and is mixed with some Gaussian observational noise), but with highly intermittent, strong-field wings (Zel'dovich et al. 1987). Simple models of this type of generation give fractal distributions (Finn \& Ott 1988). We believe that this mechanism is seen in both the QS images and in those parts of AR images where small-scale convective motions are not suppressed by strong fields.

The AR cases are more complicated. The $f(\alpha)$ curves are broader, and one discerns two distinct structures. Our interpretation is that two magnetic field generating processes are seen in the AR images. Within the ARs themselves are strong, larger-scale fields, including sunspot fields, with only small fluctuations in amplitude. These are a product of the 22 year cycle mechanism, presumably a mean field dynamo in which the field is generated by the differential rotation and mean helicity and smoothed by the turbulent diffusion (Parker 1979).

Equation (4) indicates that the Hölder exponent $\alpha_{i}$ is related to the value of the measure $\mu_{i}$ in a box. The measure is the normalized distribution of the magnitude of the line-of-sight field. The strong, sunspot-type fields will have large values of $\mu_{i}$ and thus small values of $\alpha_{i}$. Because the measure is normalized, the effect of the strong fields is to steal measure from the quietSun boxes, which leaves them with a larger Hölder exponent. Thus in Figures 4, 5, and 6, the left-hand, small $\alpha$, peak represents the large-scale field structures. The right-hand, large $\alpha$, peaks contain both Gaussian random variables and the quiet Sun, intermittent fields. The details of the interplay of the two field distributions in AR images are not yet fully understood. This will be the subject of future research.

The idea of measure, used in this paper, has both a direct observational and a deep mathematical meaning. Modern digital, photoelectric, polarimetric methods of magnetic field measurement, with CCD detectors, are surprisingly well suited to the requirements of the classical box counting approach to mathematical geometry. From the physical point of view it is necessary to cast models of magnetic field generation in the same form, so that they can be confronted with observations. We plan to find the measures predicted by some dynamo models, such as the two-dimensional random cell dynamo (Poezd, Ruzmaikin, \& Sokoloff 1992; Ruzmaikin, Liewer, \& Feynman 1993), the ABC dynamo models of Galloway \& Proctor (1992) and dynamos with a mean magnetic field. The fact that the observed measure is multifractal, with a specific form of the distribution function $f(\alpha)$ for the scaling, may seriously limit the range of possible models.

The authors acknowledge with thanks the contributions of G. A. Chapman and S. R. Walton in acquiring and processing the San Fernando Observatory data. We are grateful to K. P. Topka for providing access to the Lockheed magnetograms, to A. Brandenburg for helpful discussions, and to the anonymous referee, whose comments have led to improvement of the paper. This work was supported in part by NSF grants ATM8817634 and ATM-9115111.

\section{REFERENCES}

Balke, A. C., Schrijver, C. J., Zwaan, C., \& Tarbell, T. D. 1993, Solar Phys., 143, 215

Brandenburg, A., Procaccia, I., Segel, D., \& Vincent, A. 1992, Phys. Rev. A, 46, 4819

Bunde, A., \& Havlin, S., eds. 1991, Fractals and Disordered Systems (Berlin: Springer)

Chapman, G. A., \& Walton, S. R. 1989, in High Spatial Resolution Solar Observations, ed. O. van der Luhe (National Solar Observatory: Sunspot, NM), 402

Chhabra, A. B., Meneveau, C., Jensen, R. V., \& Sreenivasan, K. R. 1989, Phys. Rev. A, 40, 5284

Evertsz, C. J. G., \& Mandelbrot, B. B. 1992, in Chaos and Fractals: New Frontiers of Science, ed. H.-O. Peitgen, H. Jürgens, \& D. Saupe (Berlin: Springer), 921

Finn, J., \& Ott, E. 1988, Phys. Rev. Lett., 60, 760

Frisch, U., \& Parisi, G. 1985, in Turbulence and Predictability of Geophysical Flows and Climate Dynamics, ed. M. Ghil, R. Benzi, \& G. Parisi (New York: North-Holland), 84

Galloway, D. J., \& Proctor, M. R. E. 1992, Nature, 356, 691

Greimel, R., Brandt, P. N., Guenther, E., \& Mattig, W. 1990, Vistas in Astronomy, 33,413

Halmos, P. R. 1962, Measure Theory (New York: Van Nostrand)

Halsey, T. C., Jensen, M. H., Kadanoff, L. P., Procaccia, I., \& Shraiman, B. I. 1986, Phys. Rev. A, 33, 1141

Heck, A., \& Perdang, J. M., eds. 1991, Applying Fractals in Astronomy (Berlin: Springer)

Howard, R. F., \& Stenflo, J. O. 1972, Solar Phys., 22, 402

Keller, C. U. 1992, Nature, 359, 307

Lawrence, J. K. 1991, Solar Phys., 135, 249

Lawrence, J. K.. Chapman, G. A. \& Walton, S. R. 1991, ApJ, 375, 771

Lawrence, J. K., \& Schrijver, C. J. 1993, ApJ, 411, 402
Mandelbrot, B. B. 1982, The Fractal Geometry of Nature (New York: Freeman)

Mayfield, E. B., Vrabec, D., Rogers, E., Janssens, T., \& Becker, R. A. 1969 S\&T, 37,2

Meneguzzi, M., Frisch, U., \& Pouquet, A. 1981, Phys. Rev. Lett., 47, 1060

Meneveau, C., \& Sreenivasan, K. R. 1989, Phys. Lett. A, 137, 103

- 1991, 1991, J. Fluid Mech., 224, 429

Ott, E., Du, Y., Sreenivasan, K. R., Juneja, A., \& Suri, A. K. 1992, Phys. Rev. Lett., 69, 2654

Parker, E. N. 1979, Cosmical Magnetic Fields (Oxford: Clarendon)

Poezd, A. D., Ruzmaikin, A. A., \& Sokolov, D. D. 1992, Soviet Phys.-JETP Lett., 74,454

Rabin, D. 1992, ApJ, 390, L103

Ruzmaikin, A. A., Liewer, P., \& Feynman, J. 1993, Geophys. Astrophys. Fluid Dyn., in press

Ruzmaikin, A. A., Sokoloff, D. D., \& Tarbell, T. D. 1991, in The Sun and Cool Stars: Activity, Magnetism, Dynamos, ed. I. Tuominen, D. Moss, \& G. Rüdiger (Berlin: Springer), 140

Schrijver, C. J., Zwaan, C., Balke, A. C., Tarbell, T. D., \& Lawrence, J. K. 1992, A\&A, 253, L1

Stenflo, J. O. 1989, Astron. Astrophys., 1, 3

Tarbell, T. D., Acton, S., Topka, K. P., Title, A. M., Schmidt, W., \& Scharmer, G. 1990 , BAAS, 22,878

Title, A. M., Tarbell, T. D., Topka, K. P., Ferguson, S. H., Shine, R. A., and the SOUP Team 1989, ApJ, 336, 475

Zel'dovich, Ya.B., Molchanov, S. A., Ruzmaikin, A. A., \& Sokolov, D. D. 1987 , Soviet Phys.-Uspekhi, 30, 3

Zel'dovich, Ya. B., Ruzmaikin, A. A., \& Sokoloff, D. D. 1990, The Almighty Chance (Singapore: World Scientific)

Zwaan, C. 1987, ARA\&A, 25, 83 